\title{
ON AN ALMOST PERIODIC MATHIEU EQUATION*
}

\author{
BY \\ JACOB M. ABEL \\ University of Pennsylvania
}

1. Introduction. In this paper the equation

$$
\frac{d^{2} y}{d t^{2}}+\left(a-2 \sum_{i=1}^{N} q_{i} \cos 2 \lambda_{i} t\right) y=0
$$

is studied where the variables $y$ and $t$ are real and the frequencies $\left\{2 \lambda_{j}\right\}$ are a set of linearly independent (relative to the rationals) real numbers. The coefficient of $y$ in (1.1) is thus a uniformly almost periodic function in the sense of [1], and the equation viewed as an initial value problem represents an oscillator with almost periodic parametric excitation. Solutions are obtained which are generalizations of those for the Mathieu equation [2]:

$$
\frac{d^{2} y}{d t^{2}}+(a-2 q \cos 2 \lambda t) y=0
$$

and they are classified in a manner which constitutes an $N$-fold generalization of the classifications developed in the theory of (1.2), where $N$ is the number of terms in the sum in (1.1).

This problem may be viewed as a special case of the nonlinear problems treated by Moser in [3] and [4] wherein the existence of a finite basis for the spectrum of an almost pericdic function is indicated by the use of the term "quasiperiodic" after Bohl [5].

Proceeding from the result of Favard [6] which states that the boundedness of the solutions of (1.1) is equivalent to their almost periodicity, the stability of the solutions is investigated by determining the characteristic values of $a$ in (1.1) which will yield almost periodic and hence bounded solutions. The results obtained here give explicit examples of solutions of the types which are shown to exist in [3] and [4]. By suitable adjustment of the constant $a$ viewed as a function of the parameters $\left\{q_{i}\right\}$ either an even or an odd almost periodic solution of (1.1) may be obtained whose spectrum has the base $\left\{2 \lambda_{i}\right\}$. For other values of $a$, odd and even almost periodic solutions are obtained simultaneously with a common spectrum which has a basis containing an additional frequency, $\lambda_{0}$, and which is contained in the module of $\left\{2 \lambda_{j}\right\}$ translated by the amount $\lambda_{0}$. Considerations of the arithmetic relationships among $\lambda_{0}$ and the $\lambda_{i}$ leads to the determination of the geometric properties of the $N+1$ dimensional stability surfaces $a\left(q_{i}\right)$. These surfaces generalize the well-known diagram related to (1.2) (see e.g. [2]) and their intersection with any plane $\left(a, q_{i}\right)$ is precisely the stability diagram for Mathieu's equation (1.2).

* Received January 23, 1969; revised version received March 19, 1969. This work is based on a $\mathrm{Ph} . \mathrm{D}$. dissertation in Engineering Mechanics submitted to the faculty of the University of Pennsylvania. 
2. Perturbation expansions. Proceeding formally, a perturbation expansion yielding a power series in the $\left\{q_{i}\right\}$ may be obtained by a process wherein the suppression of secular terms leads to the determination of the characteristic values of $a$ for which the fundamental solutions will have the form

$$
y_{1,2}=\exp \left( \pm i \lambda_{0} t\right) \phi( \pm t)
$$

where $\phi(t)$ is an almost periodic function with the base $\left\{2 \lambda_{i}\right\}$. The form of solution (2.1) is a generalization of the Floquet form obtained in the case of periodic parametric excitation (Hill's equation) and accordingly $i \lambda_{0}$ is termed the characteristic exponent. The invariance under time reversal of (1.1) accounts for the choice of signs in (2.1). The resulting expansions may be shown to be asymptotic in the sense of Poincare [7] and subject to restrictions of an arithmetic nature on the numbers $\left\{\lambda_{j}\right\}$ the presence of "small divisors" is overcome and the expansions are convergent.

The solution of (1.1) for vanishing $q_{i}$ becomes $\exp \left(i a^{1 / 2} t\right)$ and $\exp \left(-i a^{1 / 2} t\right)$, and $a=\lambda_{0}^{2}$ is obtained. The fundamental solutions may be combined to yield independent even and odd solutions or cosine-like and sine-like solutions

$$
\begin{aligned}
& S[t]=\left(y_{1}-y_{2}\right) / 2 i \\
& C[t]=\left(y_{1}+y_{2}\right) / 2
\end{aligned}
$$

which will prove to be more convenient for computation. For nonzero $q_{i}$ the solutions are regarded as perturbations about $\cos \lambda_{0} t$ and $\sin \lambda_{0} t$ and the relationship between $a$ and the $q_{i}$ such that the solutions are almost periodic is sought. The result on the spectrum of the solution is guaranteed by the mechanics of the expansion procedure as will be seen. For initial conditions it is required that the coefficients of $\cos \lambda_{0} t$ and $\sin \lambda_{0} t$ in the even and odd solutions respectively be equal to one. A suitable normalization may be introduced later. The dependence of $a$ on the $q_{i}$ is specified as a power series in all $q_{i}$ :

$$
\begin{gathered}
a=\lambda_{0}^{2}+\sum_{m=0}^{\infty} \sum_{r=0}^{\infty} \sum_{p=0}^{\infty} \cdots \sum_{\mu=0}^{\infty} \alpha_{m n p \ldots \mu} q_{1}^{m} q_{2}^{n} q_{3}^{p} \cdots q_{N}^{\mu} \\
(m, n, p \cdots \mu) \neq(0,0,0,0 \cdots 0)
\end{gathered}
$$

where $\lambda_{0}^{2}$ is $\alpha_{000 \cdots 0}$. Similarly, for the even solution:

$$
\begin{gathered}
y=\cos \lambda_{0} t+\sum_{m=0}^{\infty} \sum_{n=0}^{\infty} \sum_{p=0}^{\infty} \cdots \sum_{\mu=0}^{\infty} C(t)_{m n p} \ldots q_{\mu} q_{1}^{m} q_{2}^{n} q_{3}^{p} \cdots q_{N}^{\mu} \\
(m, n, p \cdots \mu) \neq(0,0,0, \cdots 0)
\end{gathered}
$$

where $\cos \lambda_{0} t$ is regarded as $C(t)_{m, \ldots n}$; corresponding steps apply to the odd solution with the only difference lying in the initial conditions. From this point on the summation convention is used where possible. Using (2.3) and (2.4) to evaluate the terms in (1.1) we obtain (the dependence of tlee $C_{m n} \ldots_{\mu}$ upon $t$ is understood in what follows):

$$
\begin{gathered}
y^{\prime \prime}=-\lambda_{0}^{2} \cos \lambda_{0} t+C_{m n p}^{\prime \prime} \mu_{\mu} q_{1}^{m} q_{2}^{n} q_{2}^{p} \cdots q_{. v}^{\mu} ;(m, n, p \cdots \mu) \neq(0,0,0,0, \cdots 0) \\
a y=\lambda_{0}^{2} \cos \lambda_{0} t+C_{m n p \cdot \mu} q_{1}^{m} q_{:}^{n} q_{3}^{n} \cdots q_{1}^{\mu} \alpha_{1 ; k} \cdots q_{1}^{i} q_{2}^{j} \cdots q_{i}^{?} \\
(m, n, p \cdots \mu) \text { and }(i, j, k \cdots \Omega) \neq(0,0,0 \cdots 0) \\
\text { simultancously. }
\end{gathered}
$$


The product of the two $N$-fold power series in (2.6) is expressed by means of the Cauchy product:

$$
\begin{aligned}
& a y=\lambda_{0}^{2} \cos \lambda_{0} t+\alpha_{m-i n-i p-k} \cdots_{\mu-\Omega} C_{i j k \Omega} \ldots q_{1}^{m} q_{2}^{n} q_{3}^{p} \cdots q_{N}^{\mu} \\
& i=0,1 \cdots m \quad m=0,1, \cdots \infty \\
& j=0,1 \cdots n \quad n=0,1, \cdots \infty \quad(m, n, p \cdots \mu) \neq(0,0,0, \cdots 0) \\
& k=0,1 \cdots p \quad p=0,1, \cdots \infty \\
& \Omega=0,1 \cdots \mu \quad \mu=0,1, \cdots \infty \\
& \sum_{i=1}^{N}\left(-2 q_{i} \cos 2 \lambda_{j} t\right) y=-2 \sum_{i=1}^{N} q_{i} \cos \left(2 \lambda_{i} t\right) \cos \lambda_{0} t-2 \cos \left(2 \lambda_{1} t\right) C_{m n} \ldots \mu q_{1}^{m+1} q_{2}^{n} \cdots q_{N}^{\mu} \\
& -2 \cos \left(2 \lambda_{2} t\right) C_{m n} \ldots \mu q_{1}^{m} q_{2}^{n+1} \cdots q_{N}^{\mu} \cdots \\
& -2 \cos \left(2 \lambda_{N} t\right) C_{m n} \ldots \mu q_{1}^{m} q_{2}^{n} \cdots q_{N}^{\mu+1} .
\end{aligned}
$$

Inserting (2.5), (2.6), (2.7) and (2.8) in the differential equation and equating coefficients of each term in $q_{1} q_{2} \cdots q_{N}$ to zero yields, for example:

$$
\begin{gathered}
-\lambda_{0}^{2} \cos \lambda_{0} t+\lambda_{0}^{2} \cos \lambda_{0} t \equiv 0 \\
C_{100 \cdots 0}^{\prime \prime}+\lambda_{0}^{2} C_{100 \cdots 00}+\alpha_{100 \cdots_{0}} \cos \lambda_{0} t-2 \cos \left(2 \lambda_{1} t\right) \cos \lambda_{0} t=0 .
\end{gathered}
$$

The general term in the differential equation is

$$
\begin{aligned}
& C_{m n \mu}^{\prime \prime} \ldots+\lambda_{0}^{2} C_{m n \ldots \mu}+\alpha_{m-i n-j} \ldots_{\mu-\Omega} C_{i j \cdots \Omega} \\
& =2 \cos \left(2 \lambda_{1} t\right) C_{m-1 n \cdots \mu}+2 \cos \left(2 \lambda_{2} t\right) C_{m n-1} \cdots_{\mu} \\
& +\cdots 2 \cos \left(2 \lambda_{N} t\right) C_{m n \cdots \mu-1} \\
& (i+j+\cdots+\Omega) \geq 1 \\
& 0 \leq i<m \\
& 0 \leq j<n \\
& 0 \leq \Omega<\mu
\end{aligned}
$$

The general term is a recurrence differential equation in which the solution depends on all previously determined terms and an as yet undertermined constant $\alpha_{m n} \cdots_{\mu}$.

It will be seen in what follows that the choice of the $\alpha_{m n} \cdots_{\mu}$ is dictated by the requirement that the solution of $(1.1)$ be almost periodic. Specifically, it will be required that each term in the expansion of $y$, Eq. (2.2), be bounded for all real $t,-\infty \leq t \leq+\infty$. Inspection of (2.11) reveals that unbounded terms can arise only when the nonhomogeneous part, the forcing function, contains either unbounded, i.e. secular, terms, or periodic terms with frequency $\lambda_{0}$. Since the forcing function is derived from previously determined $C_{m n} \ldots \mu$, which are bounded even functions, the only case which can arise is that in which the forcing function contains $\cos \lambda_{0} t$. Thus, at each step in the recurrence process $\alpha_{m n} \ldots_{\mu}$ is determined so that no multiple of $\cos \lambda_{0} t$ appears in the differential equation (2.11). 
3. Classification of solutions. In carrying out the mechanics of the recursion it is found that two distinct mechanisms exist whereby a term with frequency $\lambda_{0}$ can appear. The first is more apparent, being the case wherein some product $\cos \left(2 \lambda_{j} t\right) C_{m_{1} m_{1} \cdots m_{j}-1 \cdots m N}$ upon expansion into a sum of cosines at the sum and difference frequencies gives rise to a term, $\cos \lambda_{0} t$. The second circumstance in which the frequency $\lambda_{0}$ may appear in the right-hand side of the recurrence equation occurs when some frequency $\lambda_{0}-2 m_{1} \lambda_{1}-$ $2 m_{2} \lambda_{2}-\cdots-2 m_{N} \lambda_{N}$ is equal to the number $-\lambda_{0}$, or equivalently $\lambda_{0}=$ $m_{1} \lambda_{1}+m_{2} \lambda_{2} \cdots+m_{N} \lambda_{N}$ ( $m_{i}$ integers). These considerations lead to a natural division of the characteristic solutions into classes according to whether $\lambda_{0}$ is equal to some linear combination of the $\lambda_{i}$ with positive or negative integer coefficients or whether $\lambda_{0}$ is not expressible in this manner.

When $\lambda_{0}$ satisfies some relation:

$$
\lambda_{0}=m_{1} \lambda_{1}+m_{2} \lambda_{2}+\cdots m_{N} \lambda_{N}
$$

the corresponding characteristic solution is termed a solution of integral order; when $\lambda_{0}$ does not satisfy (3.1), the characteristic solution is termed a solution of fractional order.

These designations are a natural extension of the terms used to describe the bounded solutions of the Mathieu equation, where in the canonical form the excitation has frequency 2 , and the integral and fractional solutions correspond precisely to $\lambda_{0}$ an integer or a fraction. An alternate way of representing the classification of characteristic solutions is to represent $\lambda_{0}$ as a point in an $N$-dimensional frequency space with coordinate axes corresponding to each of the $\lambda_{i}, j=1,2, \cdots, N$. The points in the space with integral coordinates are the lattice points; hence, when $\lambda_{0}$ is a lattice point the characteristic solution is of integral order and when it is not the solution is of fractional order.

4. Spectrum of solutions. By the spectrum of a function we will mean the set of frequencies appearing in the Fourier expansion of the function. As demonstrated above, the determination of the $\alpha_{m n p} \ldots \mu$ and hence of the characteristic value depends on the knowledge of the frequency spectrum of the inhomogeneous terms in the recurrence equation. The spectrum of the general term for the fractional and integral case is determined now and it will be scen that the arithmetic relationships among the frequencies are a fundamental consideration in the analysis. In this $N$-dimensional frequency space, frequencies which are linear combinations with integral coefficients of the $\left\{\lambda_{i}\right\}$ naturally are represented by the appropriate lattice points. Although fractional frequencies do not have a unique representation in this scheme, this fact causes no difficulty. A given fractional frequency, $\lambda_{0}$, may be represented by any convenient point, the. sum of whose coordinates when appropriately weighted is equal to $\lambda_{0}$. The uniqueness of the lattice point representation follows from the linear independence of the $\left\{\lambda_{i}\right\}$. The locus of all possible representations of a given fractional frequency $\lambda_{0}$ is then the hyperplane through the set of representative points and this hyperplane contains no lattice points by definition.

Solutions of Fractional Order. From the recurrence equation (2.11) it may be seen that the spectrum of any component $C_{m n} \ldots \mu$ is derived from the spectrum of the previously determined $C_{i j} \ldots \Omega$. The spectrum of each $C_{m n} \ldots_{\mu}$ is the result of a transformation of the set of points which represents the spectra of all the $C_{i j} \ldots \Omega$ appearing in (2.11) with nonzero coefficients, that is all $C_{i j} \ldots \Omega$ for which $\alpha_{m-i n-j \cdots \mu-\Omega}$ are nonzero. The multiplication of $C_{m-1 n} \cdots_{\mu}$ by $\cos 2 \lambda_{1} t$ is regarded as a transformation of the spectrum of 
$C_{m-1 n} \cdots \mu$ in which each frequency point is mapped into the pair of points $2 \lambda_{1}$ units along the $\lambda_{1}$ axis on either side of the original point. Using the symbol SP ( ) to denote "spectrum of", the spectrum of $M\left(C_{m n \cdot \mu}\right)$ before the deletion of $\lambda_{0}$ is represented as:

$$
\begin{gathered}
\operatorname{SP}\left(M\left(C_{m n} \cdots_{\mu}\right)\right)=\operatorname{SP}\left(C_{i i} \cdots_{\Omega} \mid \alpha_{m-i n-i} \cdots_{\mu-\Omega} \neq 0\right) \\
\cup \operatorname{SP}\left(C_{m-1 n} \cdots_{\mu} \cos 2 \lambda_{1} t\right) \\
\cup \operatorname{SP}\left(C_{m n-1} \cdots_{\mu} \cos 2 \lambda_{2} t\right) \\
\cup \operatorname{SP}\left(C_{m n} \cdots_{\mu-1} \cos 2 \lambda_{N} t\right) \\
\cup \operatorname{SP}\left(\alpha_{m n} \cdots_{\mu} \cos \lambda_{0} t\right) .
\end{gathered}
$$

It may be seen that the presence of $\lambda_{0}$ in any $\mathrm{SP}\left(C_{m_{1} m_{2} \cdots m_{f_{-1}} \cdots m_{N}} \cos 2 \lambda_{j} t\right)$ will require that $\alpha_{m n} \cdots_{\mu}$ be nonzero. By tracing the motion of the point $\lambda_{0}$ in the $\left\{\lambda_{j}\right\}$ space under the application of successive transformations the spectrum of the general term and the precise conditions for the establishment of nonzero $\alpha_{m n} \cdots_{\mu}$ are determined.

The spectrum of $C_{m n} \cdots \mu$ may be viewed as the result of $m$ transformations of $\lambda_{0}$ in the $\lambda_{1}$ direction and $n$ transformations in the $\lambda_{2}$ direction and so on. The reappearance of $\lambda_{0}$ in the transformed spectrum occurs whenever the number of transformations in all directions is even, i.e. $(m, n, \cdots \mu)=(2 p, 2 l, \cdots 2 t)$, with the result then that $\alpha_{m n} \cdots_{\mu}=0$ unless $m, n, \cdots \mu$ are all even. It may be concluded then that the characteristic value for solutions of fractional order is an even function of all $q_{i}$ :

$$
a=\lambda_{0}^{2}+\alpha_{2000} q_{1}^{2}+\alpha_{02 \cdots_{0}} q_{2}^{2}+\cdots \alpha_{00 \cdots_{2}} q_{N}^{2}+\alpha_{220 \cdots_{0}} q_{1}^{2} q_{2}^{2} \cdots .
$$

Further consideration of the relationship between the order of the solution and the symmetry of the characteristic values is set aside until after the establishment of the general relationships governing the spectrum. In what preceded, one mechanism for the introduction of $\lambda_{0}$ in SP $\left(C_{m n} \cdots_{\mu}\right)$ was considered. It is easily shown that the second mechanism, the introduction of $-\lambda_{0}$ in the spectrum, is excluded in the fractional case by showing that the assumption that $-\lambda_{0}$ does appear at some stage in the recurrence process for a fractional solution leads to a contradiction. Assume that

$$
\mathrm{SP}\left(C_{m n} \cdots_{\mu}\right)=\left\{-\lambda_{0} \text { and other frequencies }\right\} .
$$

This assumption implies that there are integers $p \leq m, l \leq n, \cdots, t \leq \mu$ such that

$$
\lambda_{0} \pm 2 p \lambda_{1} \pm 2 l \lambda_{2} \pm \cdots 2 t \lambda_{N}=-\lambda_{0} .
$$

However, the relation in (4.4) leads to the representation of $\lambda_{0}$ as a linear combination with integral coefficients of the $\left\{\lambda_{i}\right\}$ :

$$
\lambda_{0}=\mp p \lambda_{1} \mp l \lambda_{2} \mp \cdots \mp t \lambda_{N}
$$

which is excluded by the assumption that the solution is of fractional order. In terms of the geometric representation, it may be stated simply that no combination of transformations generated by the recurrence process exists which will map a point on the hyperplane $\lambda_{0}$ into any point on the hyperplane $-\lambda_{0}$.

Returning to the expression for the spectrum of $M\left(C_{m n} \cdots_{\mu}\right)$, Eq. (4.1) consider the term, SP $\left(C_{i j k \cdots_{\Omega}} \mid \alpha_{m-i n-i} \cdots_{\mu \cdot \Omega} \neq 0\right)$. The result on the conditions for nonzero $\alpha_{m n} \cdots_{\mu}$ is applied to ascertain which $C_{i j k \cdots \Omega}$ contribute to the spectrum of $C_{m n} \cdots \mu$. It was found earlier that the nonzero $\alpha_{m n} \cdots_{\mu}$ were those for which all indices were even; thus the 
contributing $C_{i j k \Omega}$ will be those for which $m-i, n-j, \cdots \mu-\Omega$ are even numbers greater than or equal to zero.

$$
\begin{array}{ll}
m-i=2 p & p=0,1,2 \cdots \\
n-j=2 r & r=0,1,2 \ldots \\
\vdots & \\
\mu-\Omega=2 s & s=0,1,2 \cdots .
\end{array}
$$

In essence then, the contributing $C_{i j k} \cdots_{\Omega}$ are those whose indices have the same parity as $m, n, \mu$ or (using the notion of congruence) for which the ordered $N$-tuple $(i, j \cdots \Omega)$ is congruent to $(m, n \cdots \mu)$ modulo $(2,2, \cdots 2)$.

It will be convenient henceforth to utilize the equivalence relation for ordered $N$-tuples of integers whereby $\left(m_{1}, m_{2}, \cdots m_{N}\right)$ is equivalent $(\sim)$ to $\left(n_{1}, n_{2} \cdots n_{N}\right)$ if $m_{i} \bmod 2=$ $n_{i} \bmod 2, i=1,2 \cdots N$; that is, if each element of an $N$-tuple has the same parity as the corresponding element of the sccond $N$-tuple. It is easily shown that this relation is indeed an equivalence relation and that it generates a homomorphic mapping under arithmetic mod 2 of all integer $N$-tuples onto the set of all possible $N$-tuples with 0 and 1 entries. With this foregoing as background the general result for the spectrum of $C_{m n} \cdots \mu$ may be stated. The result may be proved by induction (see [8] for details).

$$
\begin{aligned}
& \operatorname{SP}\left(C_{m n} \cdots \mu\right)=\left\{\lambda_{0} \pm 2 l \lambda_{1} \pm 2 r \lambda_{2} \pm \cdots 2 \Omega \lambda_{N}\right\} \\
& l \leq m \\
& r \leq n \quad \text { and } \quad(l, r, \cdots \Omega) \sim(m, n, \cdots \mu) . \\
& \mu \leq \Omega
\end{aligned}
$$

As a byproduct it has also been established that

$$
\alpha_{m n} \cdots \mu=0 \quad \text { unless } \quad(m, n, \mu) \sim(0,0, \cdots 0) .
$$

From these results the expression for the spectrum of the complete solution becomes:

$$
\begin{aligned}
\operatorname{SP}\left(y\left(a_{\lambda_{0}}, q_{i}, t\right)\right)= & \left\{\lambda_{\circ} \pm 2 l \lambda_{1} \pm 2 r \lambda_{2} \pm \cdots \pm 2 \Omega \lambda_{N}\right\} \\
& l=0,1 \cdots \infty \\
r & =0,1 \cdots \infty \\
& \vdots \\
& \Omega=0,1 \cdots \infty .
\end{aligned}
$$

It may be seen then that the spectrum of $y$ consists of the module generated by the $\left\{2 \lambda_{i}\right\}$ translated by an amount, $\lambda_{0}$.

Solutions of integral order. The cases in which $\lambda_{0}$ is equal to some linear combination of the $\left\{\lambda_{i}\right\}$ with integer coefficients may be classified by the parity equivalence relation. If $\lambda_{0}$ is a lattice point in the space, $\left\{\lambda_{j}\right\}$ i.e.:

$$
\lambda_{0}=m_{1} \lambda_{1}+m_{2} \lambda_{2}+m_{3} \lambda_{3} \cdots m_{N} \lambda_{N}
$$

then it may be placed into one of the clases generated by the relation $\sim$ :

$$
\lambda_{0} \varepsilon\left(m_{1} \bmod 2, m_{2} \bmod 2, m_{3} \bmod 2 \cdots m_{N} \bmod 2\right) \text {. }
$$


The representative elements for each class are the $N$-tuples with only zero or one entries, and there are $2^{N}$ of them. The classes may be ordered in accordance with the integers for which the $N$-tuple gives the binary expansion. For example, if

$$
\lambda_{0}=\left(\lambda_{1}+\lambda_{2}\right)
$$

then

$$
\lambda_{0} \varepsilon(1,1,0,0, \cdots 0)
$$

and the $N$-tuple in (4.13) is the representative of the $\left(2^{N-1}+2^{N-2}\right)$ th class. If $\lambda_{0}$ is equal to a sum of even multiples of the $\lambda_{i}$ then:

$$
\lambda_{0} \varepsilon(0,0,0,0, \cdots 0) .
$$

This $N$-tuple naturally is the zero class and defines characteristic frequencies of generalized even order. It will be shown later that the characteristic solutions of generalized even order have special properties which are common to the Mathieu functions of even order.

Spectrum of Solutions. If $\lambda_{0}$ is given by (4.10), then the considerations of the recurrence process, Eq. (2.11), are altered but not however in the early stages. Viewing the recurrence as a transformation of the spectrum commencing with $\lambda_{0}$ it may be seen that the point $-\lambda_{0}$ is not attained until the $\left(m_{1}, m_{2}, m_{3} \cdots m_{N}\right)$ th stage, that is at the determination of $C_{m_{1} m_{2} m_{3} \cdots m_{N}}$. Therefore the results for solutions of fractional order obtain for $C_{m n \cdots \mu}$ when

$$
\begin{array}{cl} 
& m<m_{1} \\
\text { or } & n<m_{2} \\
\vdots & \vdots \\
\text { or } & \\
\vdots & \mu<m_{N}
\end{array}
$$

and $\alpha_{m n} \cdots_{\mu} \neq 0$ for $(m, n, \cdots \mu) \sim(0,0, \cdots 0)$. At the $\left(m_{1}, m_{2}, \cdots m_{N}\right)$ th stage since the frequency $-\lambda_{0}$ will appear in the right-hand side of (2.11) it becomes necessary to determine a nonzero $\alpha_{m_{1} m_{2} \cdots m_{N}}$ to suppress the secular term. From that point on the frequency $-\lambda_{0}$ will be attained whenever the number of transformations in each direction subsequent to that stage is an even number. Thus, when none of the conditions (4.15) is satisfied:

$\alpha_{m n \cdots_{\mu}} \neq 0 \quad$ for $\quad\left(m-m_{1}, n-m_{2}, \cdots \mu-m_{N}\right) \sim(0,0,0, \cdots 0)$.

Thus, two sets of nonzero $\alpha_{m n} \cdots_{\mu}$ must be considered in the integral case, with the second set entering at a stage determined by the coordinates of the lattice point. This result leads to the introduction of odd powers of the $\left\{q_{j}\right\}$ in the expansion for the characteristic nuriber (except when $\lambda_{0}$ is of generalized even order). For example, if $\lambda_{0}=3 \lambda_{1}$ and $N=2$ :

$a=\lambda_{0}^{2}+\alpha_{20} q_{1}^{2}+\alpha_{02} q_{2}^{2}+\alpha_{30} q_{1}^{3}+\alpha_{40} q_{1}^{4}+\alpha_{22} q_{1}^{2} q_{2}^{2}+\alpha_{04} q_{2}^{4}+\alpha_{32} q_{1}^{3} q_{2}^{2}+\cdots$. 
In the case of generalized even order the statement (4.16) is equivalent to (4.8) and the symmetry of the expansion of the characteristic values to all orders in the $\left\{q_{i}\right\}$ is preserved.

The determination of the spectrum is made from (4.1) using (4.16).

SP $\left(C_{m n} \cdots \omega_{\mu}\right)=\left\{\lambda_{0} \pm 2 l \lambda_{1} \pm 2 r \lambda_{2} \pm 2 \Omega \lambda_{N}\right\} \cup\left\{\lambda_{0} \pm 2 l^{\prime} \lambda_{1} \pm 2 r^{\prime} \lambda_{2} \pm 2 \Omega^{\prime} \lambda_{N}\right\}$

with

$$
\begin{aligned}
& l \leq m \\
& r \leq n \quad(l, r, \cdots \Omega) \sim(m, n, \cdots \mu) \\
& \vdots \\
& \Omega \leq \mu
\end{aligned}
$$

and

$$
\begin{aligned}
& l^{\prime} \leq m-m_{1} \\
& r^{\prime} \leq n-m_{2} \quad\left(l^{\prime}, r^{\prime}, \cdots \Omega^{\prime}\right) \sim\left(m-m_{1}, n-m_{2} \cdots \mu-m_{N}\right) \\
& \vdots \\
& \Omega^{\prime} \leq \mu-m_{N}
\end{aligned}
$$

which result may be proved by induction on each of the indices. The spectrum of the characteristic solution of integral order becomes, using (4.10) and (4.18):

$\operatorname{SP}\left(y\left(a_{m_{1} m_{2} \cdots m_{N}}, q_{i}, t\right)\right)=\left\{ \pm l \lambda_{1} \pm r \lambda_{2} \cdots \pm \Omega \lambda_{N}\right\}$

$$
\cdot(l, r, \cdots \Omega) \sim\left\{m_{1}, m_{2} \cdots m_{N}\right\}
$$

or using the equivalence notation:

$$
\operatorname{SP}\left(y\left(a_{m_{1} m_{2} \cdots m_{N}}, q_{i}, t\right)\right)=\left\{\lambda \mid \lambda \sim \lambda_{0}\right\} .
$$

5. Solution classes and characteristic values. The results on the spectrum of the components of the solution lead to the establishment of some properties of the stability surfaces in the space $\left(a, q_{i}\right)$. In particular, it will be shown that for a given characteristic frequency of fractional order, the odd and even fundamental solutions of (1.1) given by (2.2) coexist, whereas in the case of characteristic frequencies of integral order the stability surfaces divide for nonzero $\left\{q_{i}\right\}$ leading to a situation wherein either the even or the odd solution will be almost periodic. As a consequence the space $\left(a, q_{i}\right)$ will be divided into regions of stability and instability bounded by the characteristic surfaces for solutions of integral order. The regions of stability consist of those points where both fundamental solutions are almost periodic and hence bounded.

Characteristic Values of Fractionai. Order. The existence of a common characteristic value for both the odd and even solutions may be established by comparing the terms in the expansions

$$
\begin{gathered}
a=\lambda_{0}^{2}+\sum_{m=0}^{\infty} \sum_{n=0}^{\infty} \cdots \sum_{\mu=0}^{\infty} \alpha_{m n \ldots \mu} q_{1}^{m} q_{2}^{n} \cdots q_{v}^{\mu}, \\
b=\lambda_{0}^{2}+\sum_{m=0}^{\infty} \sum_{n=0}^{\infty} \sum_{\mu=1}^{\infty} \beta_{m n \ldots \mu} q_{1}^{m} q_{2}^{n} \cdots q_{. v}^{\mu} \\
(m+n+\cdots \mu) \geq 1 .
\end{gathered}
$$


The equivalent of (2.11) for the odd solution is:

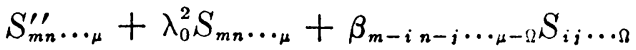

$$
\begin{aligned}
& =2 \cos 2 \lambda_{1} t S_{m-1 n \cdots \mu}+2 \cos 2 \lambda_{2} t S_{m n-1} \ldots \mu \\
& +\cdots 2 \cos 2 \lambda_{N} t S_{m n} \cdots \mu-1 \text {. }
\end{aligned}
$$

Assume that each $\alpha_{m^{\prime} n^{\prime} \cdots \mu^{\prime}}=\beta_{m^{\prime} n^{\prime} \cdots \mu^{\prime}}$ for all previously determined terms $m^{\prime} \leq m$ and $n^{\prime} \leq n$ and $\mu^{\prime} \leq \mu$ and that each $S_{m^{\prime} n^{\prime} \cdots \mu^{\prime}}$ is of the same form as the corresponding $C_{m^{\prime} n^{\prime} \cdots \mu^{\prime}}$. By this, it is meant that $S$ and $C$ have the same spectrum with equal coefficients of respective terms. Then if the multiplication of some $C_{m n} \cdots_{r-1} \cdots_{\mu}$ by $\cos 2 \lambda_{i} t$ gives rise to a term $\cos \lambda_{0} t$, the corresponding multiplication $\cos 2 \lambda_{j} t S_{m n} \cdots_{r-1} \cdots_{\mu}$ will produce a term $\sin \lambda_{0} t$ with the same coefficient. To suppress these terms it will follow that

$$
\beta_{m n p \cdots \mu}=\alpha_{m n p} \cdots \mu .
$$

It then follows that the inhomogeneous parts of (5.1) and (2.11) will be of the same form with the result that $S_{m n p \cdots \mu}$ will be of the same form as $C_{m n p \cdots \mu}$. The above statements permit the induction then that (5.2) and the appearance of terms of the same form holds for all stages in the recursion. As a consequence for $\lambda_{0}$ fractional

$$
a\left(\lambda_{0}, q_{i}\right)=b\left(\lambda_{0}, q_{i}\right)
$$

and as noted earlier they are symmetric in all $q_{i}$ to all orders.

Characteristic Values of Integral Order. When $\lambda_{0}$ is given by (4.9) the recurrence process has the character of the fractional order case until the $m_{1} m_{2} \cdots m_{N}$ th stage. At this point the frequency $-\lambda_{0}$ will appear in (2.11) and (5.1) setting the stage for the establishment of diverging values for the characteristic values $a$ and $b$. Focusing on this specific stage in the recurrence process we obtain

$$
\begin{gathered}
C_{m_{1} m_{2} \ldots m_{N}}^{\prime \prime}+\lambda_{0}^{2} C_{m_{1} m_{2} \ldots m_{N}}+\alpha_{m_{1}-i} \alpha_{m_{2}-i} \cdots_{m_{N}-\Omega} C_{i j} \cdots \Omega \\
=2 \cos 2 \lambda_{1} t C_{m_{2}-1 m_{2} \cdots m_{N}}+\cdots 2 \cos 2 \lambda_{N} t C_{m_{2} m_{2} \ldots m_{N}-1}, \\
S_{m_{1} m_{2} \cdots m_{N}}^{\prime \prime}+\lambda_{0}^{2} S_{m_{2} m_{2} \cdots m_{N}}+\beta_{m_{1}-i m_{2}-i} \cdots m_{N}-\Omega S_{i j} \cdots \Omega \\
=2 \cos 2 \lambda_{1} t S_{m_{1}-1 m_{2} \cdots m_{N}}+\cdots 2 \cos 2 \lambda_{N} t S_{m_{1} m_{2} \cdots m_{N}-1} .
\end{gathered}
$$

Since all the previously determined $\alpha_{m n} \cdots_{\mu}$ and $\beta_{m n} \cdots_{\mu}$ are equal and the previously determined $C_{m n} \ldots \mu_{\mu}$ and $S_{m n} \ldots \mu$ are of the same form, it is found that the coefficients of $\cos \left(-\lambda_{0} t\right)$ and $\sin \left(-\lambda_{0} t\right)$ in (5.4) and (5.5) are equal. The suppression of these terms then requires that

$$
\alpha_{m_{1} m_{2} \cdots m_{N}}=-\beta_{m_{1} m_{2} \cdots m_{N}}
$$

as a consequence of the oddness of $\sin \left(-\lambda_{0} t\right)$ and the evenness of $\cos \left(-\lambda_{0} t\right)$. To pursue this question further, it is necessary to determine the relationships among subsequent $\alpha_{m n} \ldots \mu$ and $\beta_{m n} \cdots \mu$ which requires in turn that the recurrence process be examined in m. ie detail. To implement this investigntion the equivalence relation, $\sim$, is employed to classify the integer $N$-tuples appearing in (2.11) and (5.1) and use is made of the arithmetic properties of the equivalence classes (i.e. their respective representatives) under addition and subtraction. The details and proof by induction of the results appear in [8] and we merely state here the findings in the form of two theorems. 
These theorems state the relationships among the $\alpha_{m n p} \cdots_{\mu}$ and $\beta_{m n p} \cdots_{\mu}$ and also define the spectra of the solution components $C_{m n p \cdots \mu}$ and $S_{m n p} \cdots_{\mu}$. As will be evident, the parity of the integers in the expression for the characteristic frequency, $\lambda_{0}$, plays a dominant role in establishing the form of the results. In addition, we recall that the results differ depending upon whether or not the recurrence process has reached the stage where the point $-\lambda_{0}$ is attainable.

Theorem I. For a given characteristic frequency of integral order $\lambda_{0}=p_{1} \lambda_{1}+$ $p_{2} \lambda_{2}+\cdots p_{N} \lambda_{N}$ with $\lambda_{0}$ in the equiralence class $P$, the solution components $C_{m n \cdots_{\mu}}, S_{m n p} \cdots_{\mu}$ and the coefficients in the expansion for the characteristic values satisfy the following relationships.

A. For: $m<p_{1}$ or $n<p_{2}$ or $p<p_{3}$ or $\mu<p_{N}$ :

1. The frequencies in $C_{m n p} \cdots_{\mu}\left(S_{m n p} \cdots_{\mu}\right)$ are in the same equivalence class as the $N$ tuple $(m, n, p \cdots \mu)$.

2. The nonzero $\alpha_{i j k} \cdots \Omega\left(\beta_{i j k} \cdots_{\Omega}\right)$ are in the class $Q$ (all elements even).

B. For $m \geq p_{1}$ and $n \geq p_{2}$ and $p \geq p_{3}$ and $\cdots \mu \geq m_{N}$ :

1. If the class of the $N$-tuple $(m, n, p \cdots \mu)$ is denoted as $Q$, then the spectrum of $C_{m n p \cdots \mu}\left(S_{m n p \cdots \mu}\right)$ is in the classes $Q$ and $Q+P$.

2. The indices of the nonzero $\alpha_{i{ }_{k} \ldots \Omega}\left(\beta_{i j k} \ldots \Omega\right)$ are in the classes $Q$ and $P$.

3. Denoting $\alpha_{m n p} \cdots \mu$ when $(m, n, p \cdots \mu)$ is in a class $Q$ by $\alpha_{0}$, the following is true:

a. For $Q=P$

$$
\alpha_{0}=-\beta_{0} \neq 0 .
$$

b. For $Q=a$

$$
\alpha_{Q}=\beta_{Q} \neq 0 .
$$

c. For $Q=P=Q\left(\lambda_{0}\right.$ generalized even order and even powered term in expansion)

$$
\alpha_{0} \neq \beta_{0}
$$

but

$$
\alpha_{o}+\beta_{o} \neq 0 .
$$

With regard to the relationship between the solution components the following is found.

Theorem II. A. For $(m, n, p \cdots \mu)$ in $Q$ and $Q \neq Q$ :

1. The coefficients of corresponding terms in $C_{m n p} \cdots_{\mu}$ and $S_{m n p} \cdots_{\mu}$ whose frequencies are in $Q$ are equal.

2. The coefficients of corresponding terms in $C_{m n n} \cdots_{\mu}$ and $S_{m n p} \cdots_{\mu}$ whose frequencies are in $Q-P$ are equal in magnitude and of opposite sign.

6. Geometry of the characteristic surfaces. From the foregoing, we see that the symmetry properties and degree of contact of characteristic surfaces of integral order depends on the specific class of the characteristic frequency, or equivalently upon the parity of cach of the integers in the expression:

$$
\lambda_{0}=\left(m_{1} \lambda_{1}+m_{2} \lambda_{2}+\cdots m_{*} \lambda_{N}\right) .
$$

In the fractional case, all the characteristic surfaces are symmetric with respect to each axis, $q_{i}$, and the characteristic values for the odd and even solutions are equal. Thus 
we may say that the surfaces for the odd and even characteristic values collapse into one surface or that their degree of contact is infinite.

In the integral case, we may group the $q_{i}$ axes according to whether $m_{i}$ in (6.1) is odd or even. We denote the two sets of axes by $O$ and $E$ respectively.

We found earlier that the characteristic surfaces for the odd and even solutions corresponding to a given $\lambda_{0}$ were in contact up to the term $q_{1}^{m_{1}} q_{2}^{m_{2}} q_{3}^{m_{3}} \cdots q_{N}^{m_{N}}$. In addition, we noted that the surfaces were symmetric up to terms of that order. Subsequent to the $m_{1} m_{2} m_{3} \cdots m_{N}$ th term, the expansion for the characteristic values $a\left(q_{i}\right), b\left(q_{i}\right)$ contains terms whose exponents are equivalent to the $N$-tuple $\left(m_{1}, m_{2}, m_{3} \cdots m_{N}\right)$. We see then that the characteristic surfaces will be completely symmetric with respect to any axis in the set $E$. Also from the relation $\alpha_{p}=-\beta_{p}$, we may deduce an interesting relationship between $a\left(q_{i}\right)$ and $b\left(q_{i}\right)$. We denote the characteristic values as functions of the two sets of $q_{i}$ explicitly to emphasize the relation:

$$
a=a\left(q_{E}, q_{0}\right), \quad b=b\left(q_{E}, q_{0}\right) .
$$

We consider the effect of inverting one axis $q_{2}$ while leaving the others unchanged. In the case $q_{i}$ in $E$ obviously

$$
a\left(q_{E}, q_{0}, q_{i}\right)=a\left(q_{E}, q_{0},-q_{i}\right)
$$

while by virtue of the relations between the $\alpha_{i j} \cdots_{\mu}$ and $\beta_{i j} \cdots_{\mu}$, we have when $q_{i}$ is in $O$ :

$$
a\left(q_{E}, q_{0}, q_{i}\right)=b\left(q_{E}, q_{0},-q_{i}\right) .
$$

Thus while the individual characteristic surfaces may not be symmetric with respect to an axis $q_{i}$, their intersections with any plane $\left(a, q_{i}\right)$ will be symmetric. This result reduces precisely to the result obtained in the case of the Mathieu equation wherein characteristic curves of even order are symmetric in $q$ and curves of odd order obey

$$
a_{0}(q)=b_{0}(-q) \text {. }
$$

We find then that the zones of stability and instability defined by the set of characteristic surfaces for the odd and even solutions of integral order are symmetric. With respect to a given pair of surfaces $a\left(q_{i}\right)$ and $b\left(q_{i}\right)$, we have shown that both surfaces are individually symmetric with respect to the $q_{i}$ in $E$, and with respect to the $q_{i}$ in $O$, the two surfaces satisfy (6.4). Thus the $n+1$ dimensional region enclosed by the pair of surfaces is symmetric with respect to all the $q_{i}$. We note one additional property of the complete stability diagram which is a consequence of the linear independence of the $\lambda_{i}$. The characteristic frequency $\lambda_{0}$ can be an integral multiple of at most one of the $\lambda_{j}$, say $\lambda_{i}$; therefore $\lambda_{0}$ must be of fractional order relative to at least $N-1$ of the $\lambda_{i}$. Thus in each of the planes $\left(a, q_{i}\right.$ ) (a Mathieu plane) for which $\lambda_{i} \neq \lambda_{i}$, the odd and even characteristic surfaces must intersect in a single curve. When $\lambda_{0}$ is not a lattice point on any one of the $\lambda_{j}$ axes, it is fractional with respect to all $N$ of the $\lambda_{j}$, and the characteristic surfaces $a\left(q_{i}\right)$ and $b\left(q_{j}\right)$ intersect every plane $\left(a, q_{i}\right)$ in a single curve. In Fig. 1, we show a sketch of the characteristic surfaces for the two-frequency case $N=2$ where $\lambda_{0}=\lambda_{1}=\pi$ and $\lambda_{2} \ldots \pi \sqrt{2}$. The surfaces intersect the $\left(a, q_{1}\right)$ plane in the Mathicu curves of order ons. The two surfaces intersect the $\left(a, q_{2}\right)$ plane in the single Mathieu curve of fractional order $\sqrt{2} / 2$. In Iig. 2, we show the intersection of these surfaces with planes for which $q_{2} / q_{1}=a$ constant.

7. Conclusions. On the basis of Favard's cited result the question of the stability of 


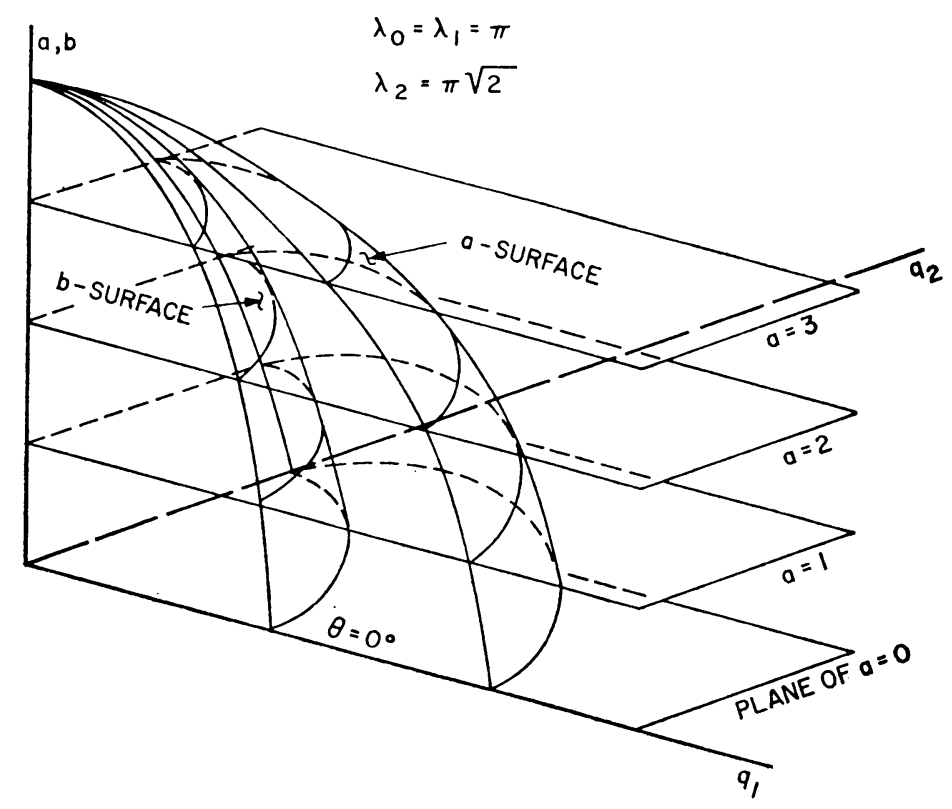

FIG. 1. Characteristic surfaces of integral order

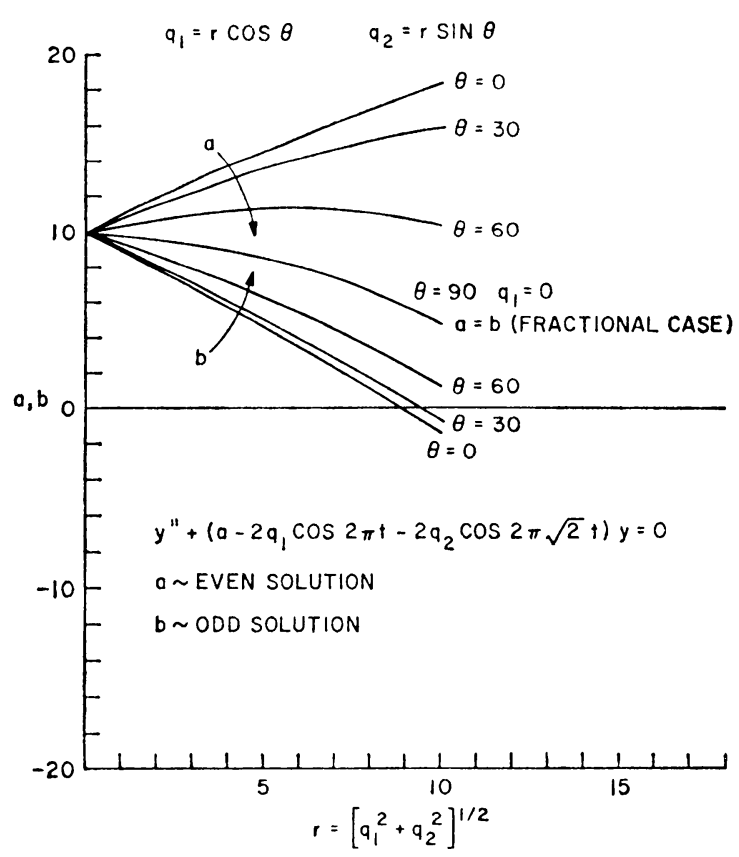

FIG. 2. Characteristic values of integral order $\lambda_{0}=\lambda_{1}=\pi, \lambda_{2}=\sqrt{2} \pi q_{1}=r \cos \theta, q_{2}=r \sin \theta$ 
(1.1) has been transformed into that of determining characteristic values $a\left(q_{i}\right)$ which will yield almost periodic solutions. The expansion procedure coupled with the classical method of suppressing secular terms has illuminated the structure of the stability diagram as well as providing the means of computing characteristic values and solutions which asymptotically satisfy (1.1). We note in particular that the results obtained here generalize those of the Mathieu theory in many respects, some others of which will be reported in a subsequent paper. The resulting stability diagram for (1.1) appears as a densely ramified set of nested hypersurfaces in the space $\left\{a, q_{i}\right\} j=1,2, \cdots N$ which enclose regions of stability in which bounded odd and even solutions exist simultaneously. The spectra of the solutions display the general proliferation of frequency content which can be achieved by parametrically exciting an oscillator with an almost periodic function.

\section{REFERENCES}

[1] A. S. Besicovitch, Almost periodic functions, Dover, New York, 1953

[2] N. McLachlan, Theory and application of Mathieu functions, Oxford Univ. Press, New York, 1947

[3] J. Moser, Combination tones for Duffing's equation, Comm. Pure Appl. Math. 18, 17-181 (1965)

[4] J. Moser, On the theory of quasiperiodic motions, SIAM Rev., 8, April (1966)

[5] P. Bohl, Sur certaines equations différentielles d'un type général utilisable méchanique, Bull. Soc. Math. France 38, 5-138 (1910)

[6] J. Favard, Leçons sur les functions presque-periodiques, Gauthier-Villars, Paris, 1933

[7] H. Poincaré, Les methodes nouvelles de la mécanique célèste, vol. II, Dover, New York, 1957

[8] J. M. Abel, The stability of dynamic and elastic systems with almost periodic or nearly periodic excitation, Ph.D. thesis, University of Pennsylvania, Philadelphia, Pa., 1966 